\title{
A Class Of Selenocentric Retrograde Orbits With Innovative Applications To Human Lunar Operations
}

\author{
Daniel R. Adamo* \\ Independent Astrodynamics Consultant, Salem, OR 97306 \\ Dr. Daniel F. Lester ${ }^{\dagger}$ \\ University of Texas, Austin, TX 78712-0259 \\ Dr. Harley A. Thronson \\ NASA Goddard Space Flight Center, Greenbelt, MD 20771 \\ and \\ Brent W. Barbee ${ }^{\S}$ \\ NASA Goddard Space Flight Center, Greenbelt, MD 20771
}

\begin{abstract}
Selenocentric distant retrograde orbits with radii from $\sim 12,500 \mathrm{~km}$ to $\sim \mathbf{2 5 , 0 0 0} \mathbf{~ k m}$ are assessed for stability and for suitability as crewed command and control infrastructure locations in support of telerobotic lunar surface operations and interplanetary human transport. Such orbits enable consistent transits to and from Earth at virtually any time if they are coplanar with the Moon's geocentric orbit. They possess multiple attributes and applications distinct from NASA's proposed destination orbit for a redirected asteroid about $70,000 \mathrm{~km}$ from the Moon.
\end{abstract}

\section{Nomenclature}

$a \quad=$ semi-major axis

$e \quad=$ orbit eccentricity

$r_{0} \quad=$ strictly, a selenocentric orbit's radius at coasted numeric integration initialization and synonymous with a stable selenocentric orbit's mean radius over time

$r_{S O I}=$ gravitational sphere-of-influence radius

$T=$ selenographic period, the time required for a selenocentric orbit's selenographic longitude to repeat

$t=$ coasted numeric integration time since initialization

$v_{\infty} \quad=$ asymptotic speed

$\Delta v \quad=$ vector difference magnitude between two velocities (change-in-velocity)

$\Delta v_{C}=\Delta v$ required to circularize an orbit

$\Delta v_{F B}=\Delta v$ applied during a flyby periapsis

\footnotetext{
* Sole proprietor, 8119 Kloshe Ct. S, AIAA Senior Member.

${ }^{\dagger}$ Research Fellow, Department of Astronomy, 1 University Station C1400.

* Senior Scientist, Advanced Concepts in Astrophysics, Code 660, 8800 Greenbelt Rd., AIAA Member.

$\S$ Aerospace Engineer, Code 595, 8800 Greenbelt Rd., AIAA Member.
} 
$\Delta v_{T O T}=$ sum of all pertinent $\Delta v$ values

$\varepsilon \quad=$ selenocentric evection angle between pericynthion and Earth's position

$\phi \quad=$ selenographic latitude

\section{Introduction}

IN association with NASA's asteroid strategy published 10 April 2013 (Ref. 1), selenocentric $\mathbf{I}_{\text {distant retrograde orbits (SDROs) have assumed a new importance in the field of astronautics. }}$ The strategy's asteroid redirect mission (ARM) concept proposes a small near-Earth asteroid (NEA) be brought to "a stable orbit in trans-lunar space". Astronauts would subsequently rendezvous with it. In its ARM concept document published 27 June 2013 (Ref. 2), NASA elaborates, "The storage orbit for the redirected asteroid is a stable distant retrograde orbit (DRO) in the Earth-Moon system with an orbit altitude of $\sim 70,000 \mathrm{~km}$ above the lunar surface."

The largest practical SDRO mean radius, $r_{0}$, is desirable in an ARM context. This criterion helps minimize change-in-velocity magnitude, $\Delta v$, necessary to achieve a stable SDRO for a redirected NEA whose mass is $\sim 500 \mathrm{mt}$ (Ref. 1). But an SDRO with $r_{0}$ considerably less than $70,000 \mathrm{~km}$ can also be stable. Only at $r_{0}<\sim 2700 \mathrm{~km}$ does an SDRO become unstable due to non-uniform lunar gravity (Ref. 3, p. 58).

Stability and utility of SDROs nearly coplanar with the Moon's geocentric orbit having radii from $\sim 12,500 \mathrm{~km}$ to $\sim 25,000 \mathrm{~km}$ are the focus of this paper. To distinguish orbits at these and adjacent distances from ARM's destination, they are termed proximal SDROs (PSDROs) hereinafter. In selenocentric architectures involving lunar surface operations, human infrastructure in a PSDRO is at a highly desirable command-and-control location. Data latency while communicating with lunar surface systems is minimal, and unobstructed line-of-sight for direct communications with a fixed surface location is uninterrupted for hours. Logistics between Earth and PSDRO-resident infrastructure are also more robust than at the $r_{0}=70,000$ $\mathrm{km}$ ARM venue because they can be conducted at virtually any departure time without large variations in transit time or $\Delta v$. This PSDRO attribute also enables resident infrastructure support of arriving and departing interplanetary human transports.

\section{Theory Governing SDRO Stability}

Numeric simulations of small moons orbiting the Jovian planets (Ref. 4) indicate escape to interplanetary space will occur in a few decades for prograde orbits with semi-major axes half the planet's gravitational sphere-of-influence radius $\left(a=0.5 r_{S O I}\right)$. For retrograde orbits, this fate is suffered only at $a>0.7 r_{S O I}$. Escapes from Jovian planets are caused by the evection resonance, in which the simulated planet-centered orbit's apoapsis aligns to a sufficient degree and frequency with direction to the Sun. This alignment increases eccentricity $e$ to unity over time and causes escape from the planet. Evection resonances occur less frequently and persist over shorter time intervals in retrograde orbits than in equivalent prograde orbits.

For this paper's SDRO context, Earth replaces the Sun as source of evection perturbations. The evection angle $\varepsilon$ is used to quantify the degree to which evection is perturbing an SDRO, as illustrated in Figure 1. 


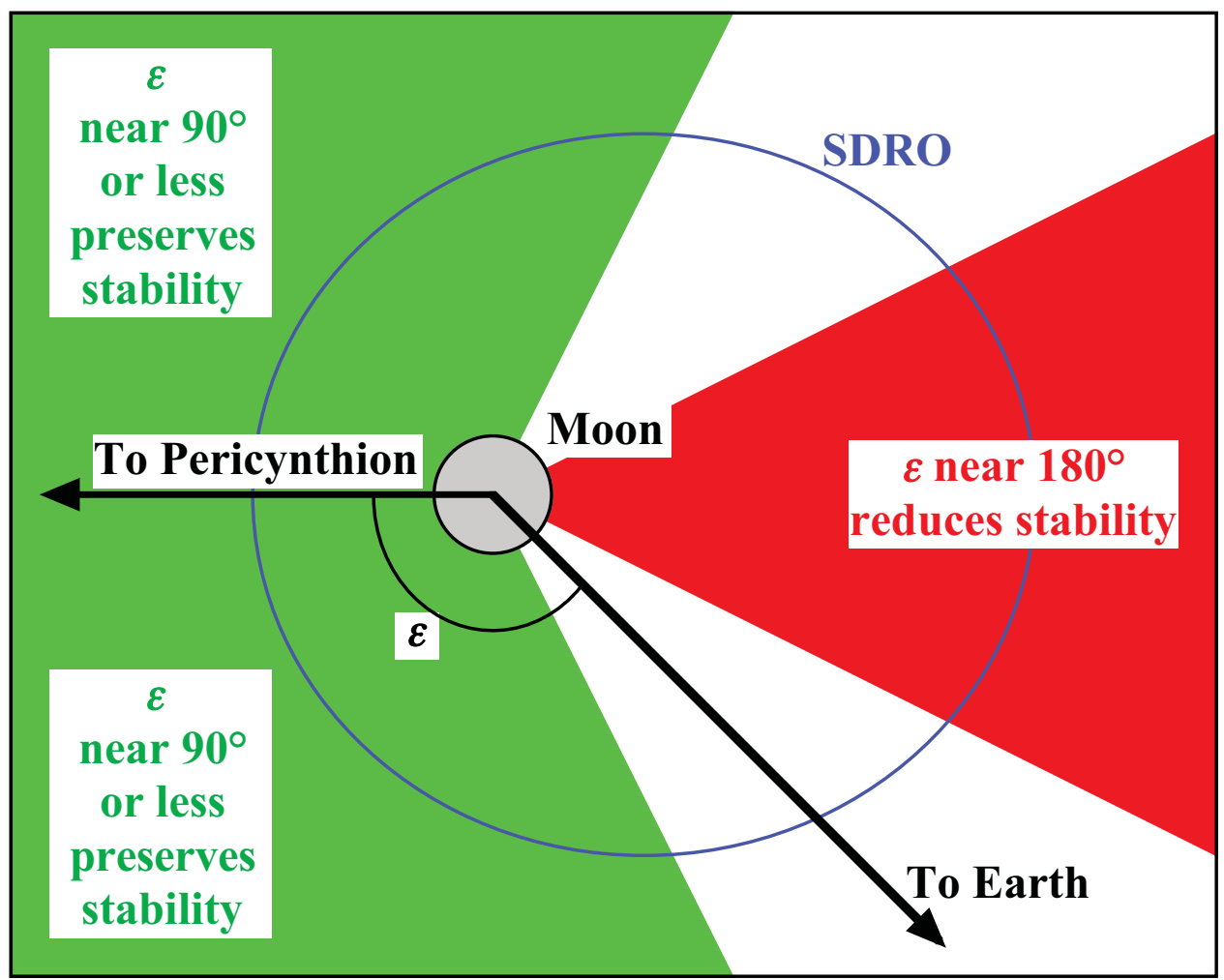

Figure 1. The evection angle $\varepsilon$ is defined for an SDRO (blue). Approximate selenocentric regions from which Earth preserves (green) or reduces (red) SDRO stability are illustrated. White selenocentric regions indicate $\varepsilon$ values whose SDRO stability effects are marginal.

An empirical orbit stability definition is adopted herein where initial conditions are coasted through time $t$ using numeric integration of point-mass gravitational accelerations from the Moon, Earth, and Sun. To ensure well-converged numeric integration at a fixed $600 \mathrm{~s}$ step size, any such coast is deemed unstable if it reaches a selenocentric radius less than $4300 \mathrm{~km}$. At this distance, selenocentric escape speed is approximately equal to speed in a selenocentric circular orbit with $r_{0}=2160 \mathrm{~km}$, about $1.5 \mathrm{~km} / \mathrm{s}$. Integration through one such circular orbit requires 15 of the adopted $600 \mathrm{~s}$ steps and produces well-converged results (Ref. 5).

Over coasting intervals as long as 100 years, SDRO stability is equated with the degree to which selenocentric distance is confined. Therefore, a coast exceeding $2 r_{S O I}$ (in practice, $140,000 \mathrm{~km}$ selenocentric distance) is also deemed unstable.

With the Moon's $r_{S O I}=66,000 \mathrm{~km}$ (Ref. 6, p. 4), consider a PSDRO and its prograde equivalent at $r_{0}=25,000 \mathrm{~km}=0.38 r_{S O I}$. Figures 2 and 3 plot $\varepsilon$ and $e$ as functions of numeric integration coast time $0 \leq t \leq 12$ years for the PSDRO and its prograde equivalent, respectively. 


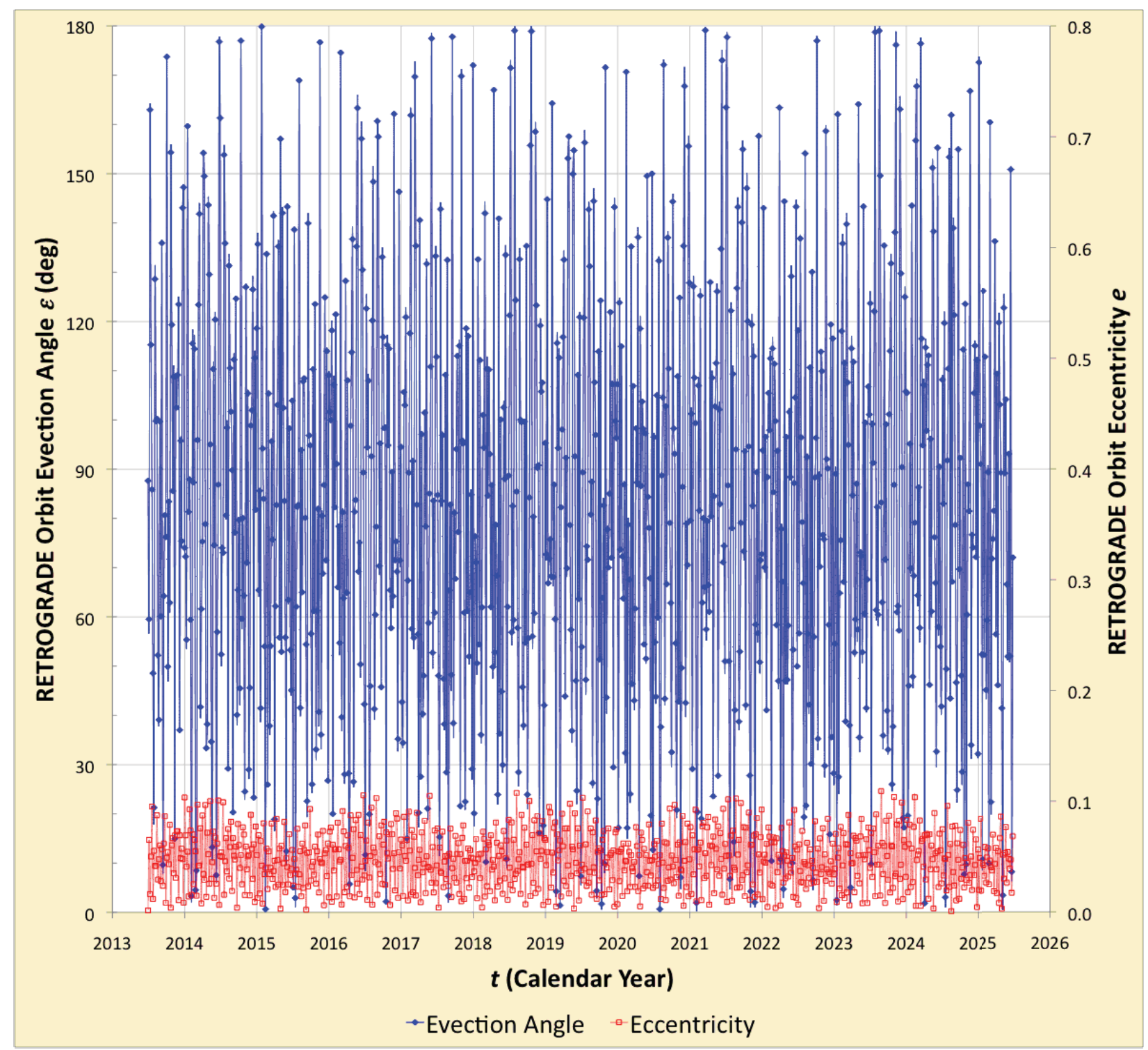

Figure 2. Variations in evection angle $\varepsilon$ (filled blue diamonds; scale at left) and eccentricity $e$ (unfilled red squares; scale at right) are plotted for a PSDRO with $r_{0}=\mathbf{2 5 , 0 0 0} \mathbf{~ k m}$ as a function of numeric integration coast time $t$. The $e$ scale is extended to match that in Figure 3 and thereby facilitate comparisons. 


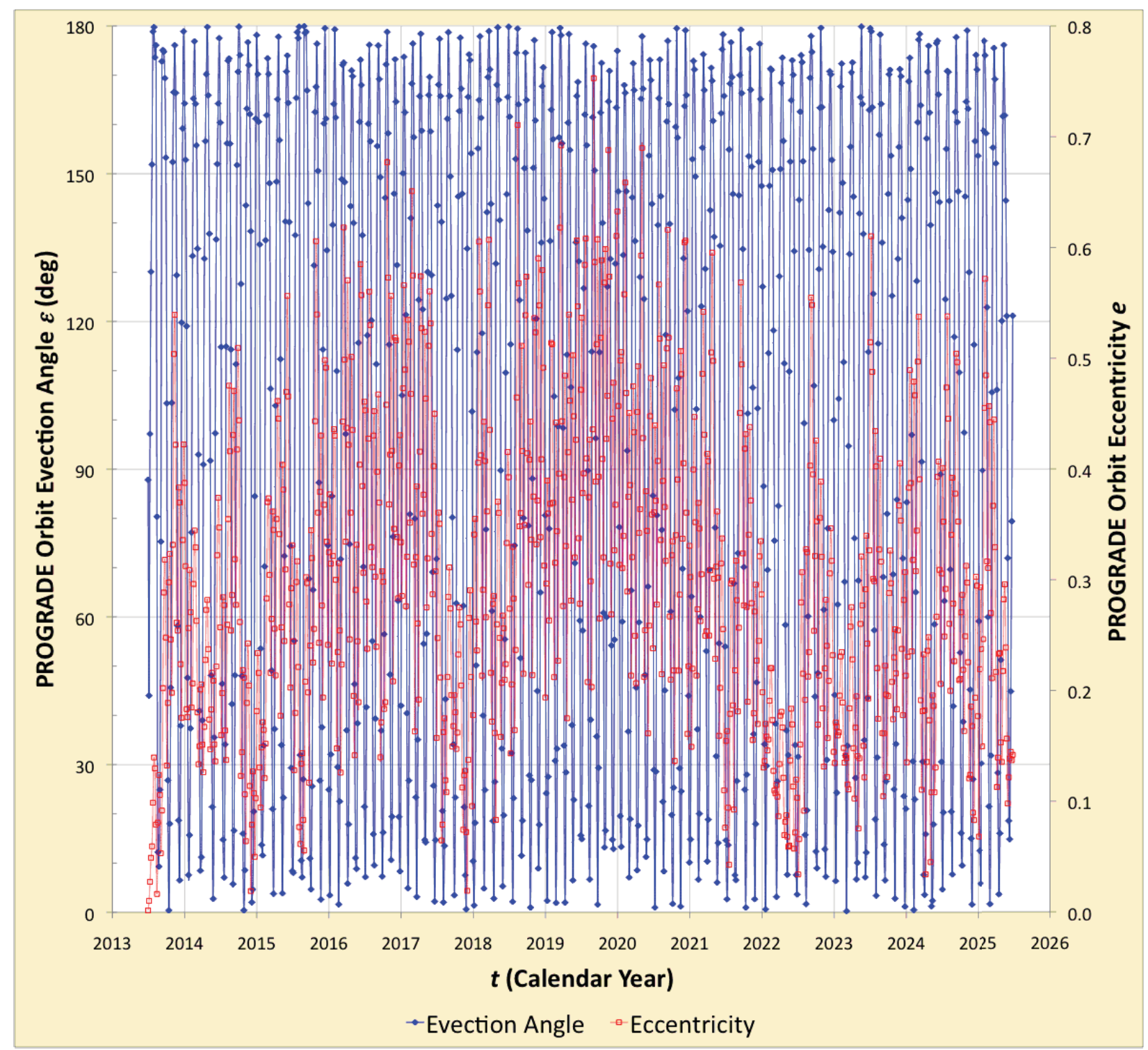

Figure 3. Variations in evection angle $\varepsilon$ (filled blue diamonds; scale at left) and eccentricity $e$ (unfilled red squares; scale at right) are plotted for the prograde equivalent of Figure 2.

Even visual inspection indicates clustering of $\varepsilon$ points in Figure 2 is more toward $90^{\circ}$ than in Figure 3. Note also the smaller $e$ variations in Figure 2 with respect to Figure 3. Both of these metrics indicate the PSDRO enjoys enhanced stability with respect to its prograde counterpart.

To quantify $\varepsilon$ distributions in Figures 2 and 3, the 877 data points in each dataset are sorted into bins $30^{\circ}$ wide, and a tally is made of each bin's data points. Results of these tallies appear in Table 1 and confirm evection theory's prediction the PSDRO is more stable than its prograde counterpart. Despite these relative statistics, the dynamic nature of $\varepsilon$ evident in Figures 2 and 3 renders this metric a necessary, but not sufficient condition for orbit stability as defined in this paper. 
Table 1. The degree to which certain ranges (bins) in evection angle $\varepsilon$ are populated during 12-year coasts is quantified with tallies from the 877 data points in Figure 2 (Retrograde) and the 877 data points in Figure 3 (Prograde) at $r_{0}=25,000 \mathrm{~km}$. Greater numbers of $\varepsilon$ points clustered near $90^{\circ}$ is a condition conferring enhanced stability on the retrograde orbit with respect to its prograde equivalent.

\begin{tabular}{|c|c|c|}
\hline$\varepsilon$ Bin (deg) & Retrograde Tally & Prograde Tally \\
\hline 0 to 30 & 108 & 210 \\
\hline 30 to 60 & 160 & 105 \\
\hline 60 to 90 & 227 & 76 \\
\hline 90 to 120 & 203 & 66 \\
\hline 120 to 150 & 109 & 123 \\
\hline 150 to 180 & 70 & 297 \\
\hline
\end{tabular}

Conclusive confirmation of the $r_{0}=25,000 \mathrm{~km}$ PSDRO's stability and its prograde counterpart's instability is evident in selenocentric inertial plots of coasted motion projected into the ecliptic plane. Figure 4 is the PSDRO's plot over $t=100$ years, and Figure 5 is the prograde equivalent over $t=12$ years. Note Figure 5's scale is identical to Figure 4's.

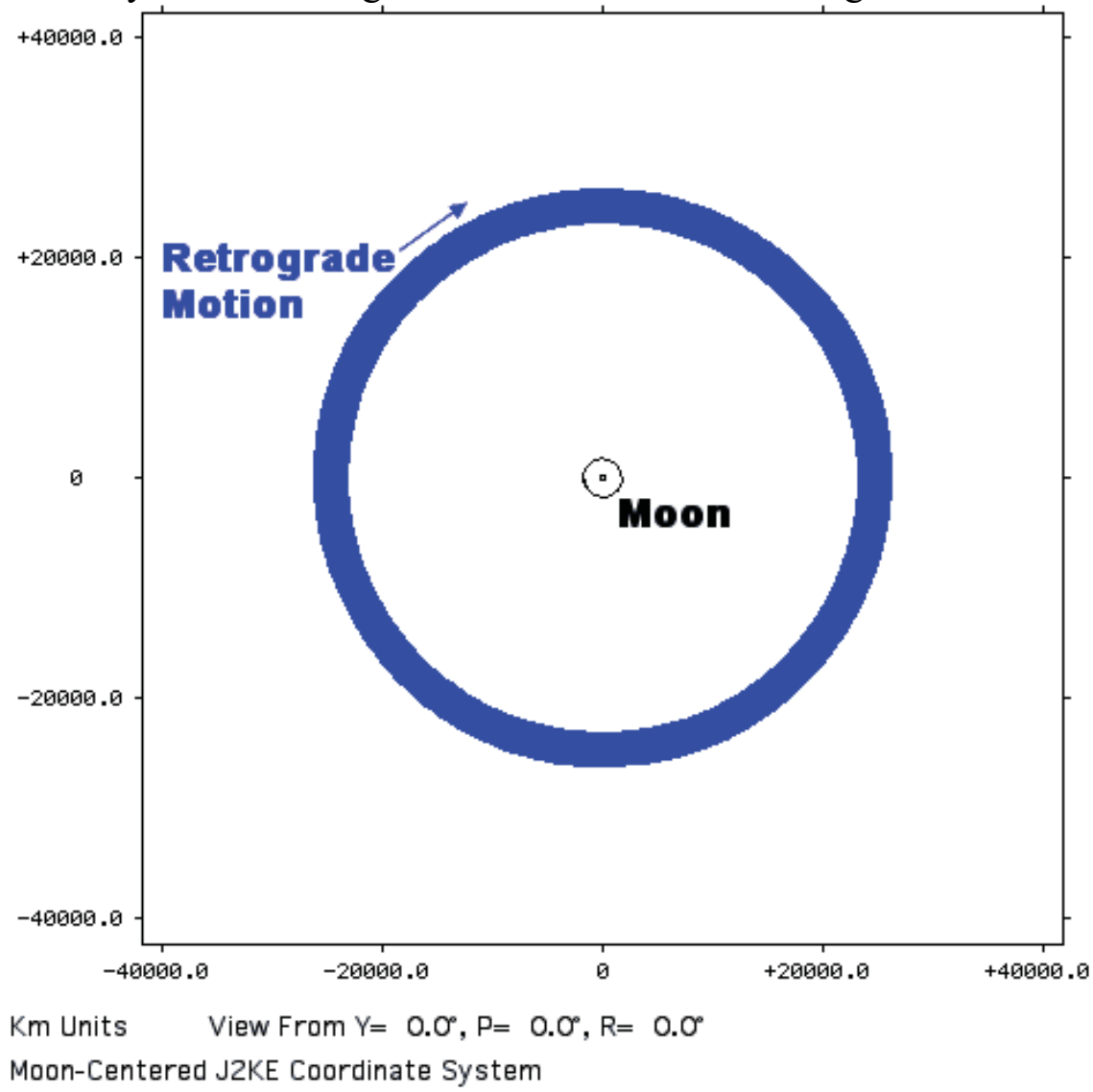

Figure 4. Coasted inertial selenocentric motion of a PSDRO at $\boldsymbol{r}_{0}=\mathbf{2 5 , 0 0 0} \mathbf{k m}$ is plotted in the ecliptic plane for 100 years. This motion is tightly confined to a narrow annular region and qualifies as stable. The smaller black selenocentric circle circumscribes the Moon's north rotational pole. 


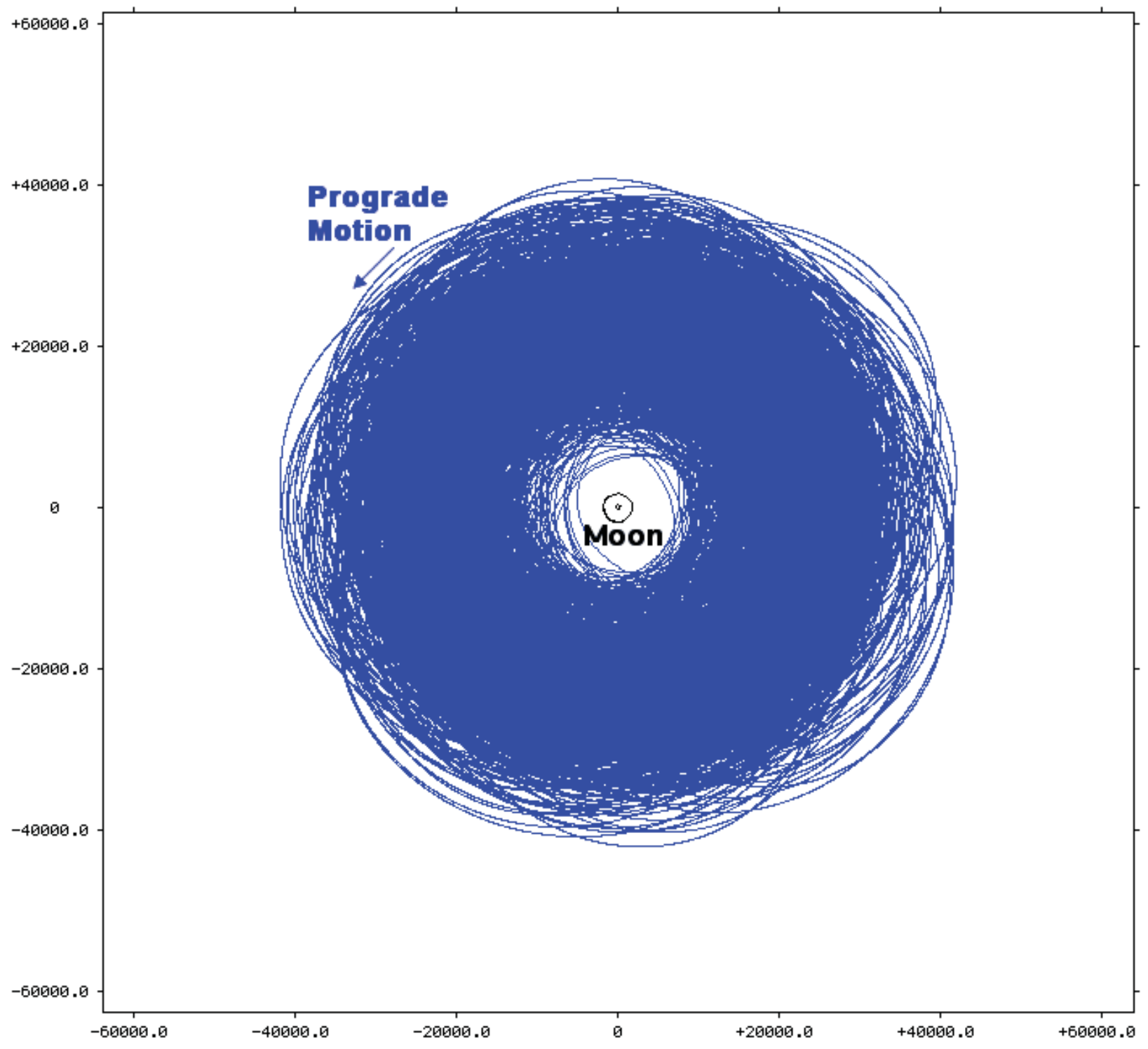

Km Units View From $Y=0.0^{\circ}, P=0.0^{\circ}, R=0.0^{\circ}$

Moon-Centered J2KE Coordinate System

Figure 5. Coasted inertial selenocentric motion, initially the prograde equivalent to Figure 4, is plotted at Figure 4's scale in the ecliptic plane for 12 years. This motion becomes eccentric and qualifies as unstable because selenocentric distance falls below $4300 \mathrm{~km}$ after coasting 28.1 years. The smaller black selenocentric circle circumscribes the Moon's north rotational pole.

\section{PSDRO Attributes}

The following subsections relate PSDRO attributes relevant to selenocentric human operations. A majority of these attributes substantiate PSDROs as a highly desirable location for human command and control of lunar surface assets, while also serving as a garage for human interplanetary transports. 


\section{A. Selenographic Period}

Assuming unperturbed selenocentric conic motion, selenographic period $T$ reflects the sum of lunar angular rotational rate and SDRO angular rate. Conic $T$ values are plotted as a function of $r_{0}$ by the solid blue curve with diamond markers in Figure 6. Co-plotted as red square markers in Figure 6 are $T$ values inferred from numeric integration coasts at $r_{0}=12,500 \mathrm{~km}, 25,000 \mathrm{~km}$, and $70,000 \mathrm{~km}$. Coast-derived markers indicate the increasing degree to which SDROs depart from conic motion as $r_{0}$ increases to exceed $r_{S O I}$.

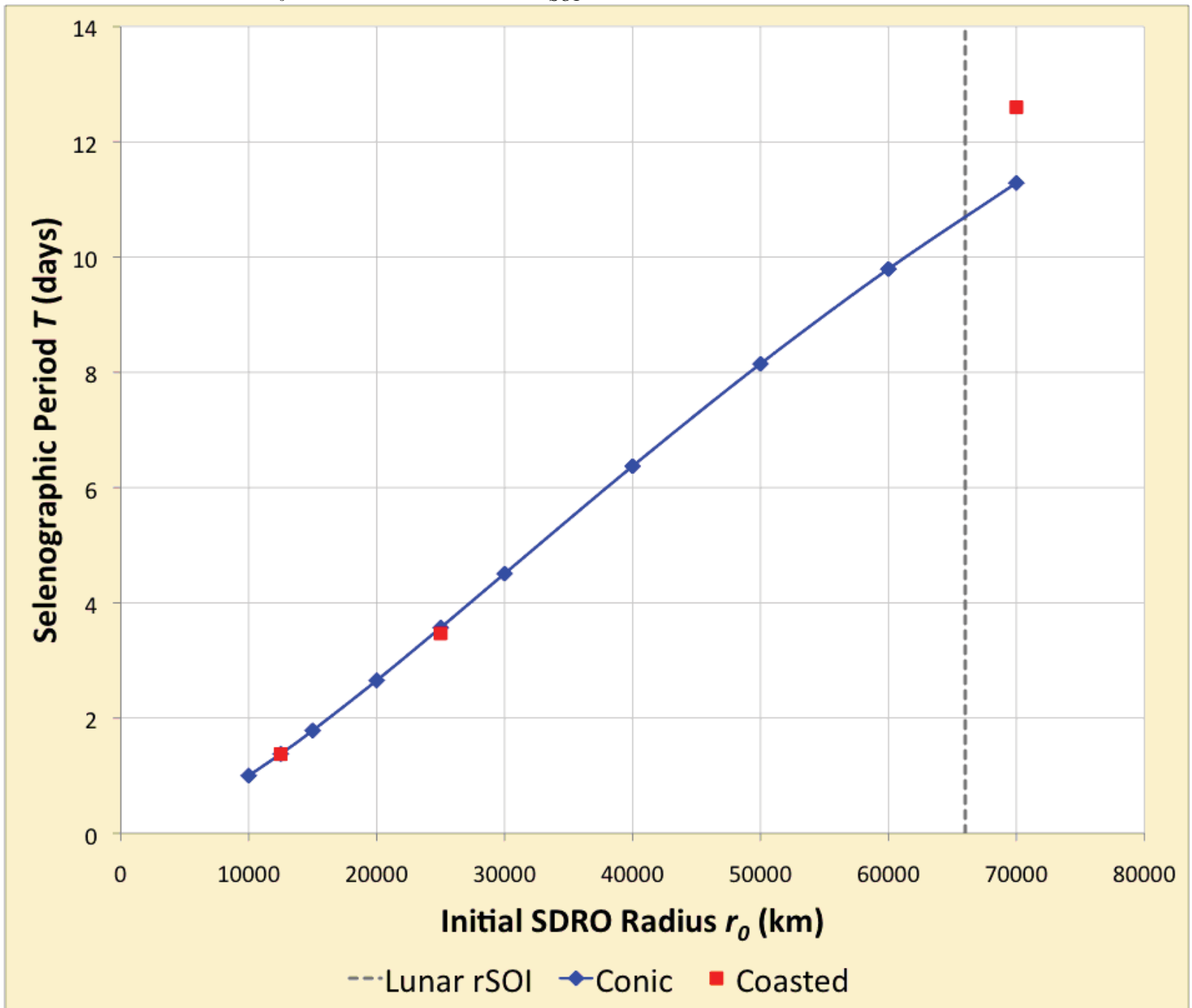

Figure 6. Selenographic period $T$ is plotted as a function of $r_{0}$ assuming unperturbed conic motion (solid blue curve with diamond markers) and coasted numeric integration of Moon, Earth, and Sun gravity accelerations (red square markers).

Transits between Earth and infrastructure in an SDRO will tend to cycle through variations in $\Delta v$ during $T$. This relationship arises in part because selenographic longitude is nearly fixed over time with respect to the Earth/Moon line and with respect to directions of $\Delta v$-minimal retrograde approach or departure tangent to the SDRO. Variations in Earth/SDRO infrastructure transit time may be imposed to reduce $\Delta v$ below unacceptable levels in otherwise non-optimal cases. This technique is subject to human factors and consumables limitations with a crew aboard, but 
transit time variations amounting to a day or two are typically available at any departure epoch ${ }^{* *}$. If any-day Earth departure capability is a program requirement, Figure 6 therefore indicates a nearly optimal rendezvous with SDRO-resident infrastructure can be achieved for $r_{0}<16,000$ $\mathrm{km}$ since $T<2$ days under this condition.

In a broader context, PSDROs lie deeper in the Moon's gravity well than the $r_{0}=70,000 \mathrm{~km}$ SDRO proposed for ARM. In cases with optimal timing, transits between Earth and a redirected asteroid at $r_{0}=70,000 \mathrm{~km}$ will require less $\Delta v$ than any transit between Earth and infrastructure at $r_{0}<16,000 \mathrm{~km}$. But optimal cases at $r_{0}=70,000 \mathrm{~km}$ may be separated by nearly 2 weeks, as indicated by Figure 6, with significantly higher transit $\Delta v$ requirements in the interim. At $r_{0}<$ $16,000 \mathrm{~km}$, transit $\Delta v$ variations can be virtually eliminated, greatly simplifying any-time Earth logistics. Similar Earth-transit variations associated with infrastructure in a periodic orbit about the cislunar libration point (EML1) have been identified (Ref. 7, Section 3.3).

A beneficial consequence of near-constant Earth logistics $\Delta v$ constraining $r_{0}<16,000 \mathrm{~km}$ is communications data latencies no more than one-quarter those experienced at $r_{0}=70,000 \mathrm{~km}$. With PSDRO infrastructure only $12,500 \mathrm{~km}$ from a surface asset, round-trip command/response verification would be carried out in $0.08 \mathrm{~s}$. Transport time between orbiting infrastructure and lunar surface assets would likewise be reduced. For example, a conic Hohmann transfer from $r_{0}$ $=70,000 \mathrm{~km}$ to the lunar surface requires over $84 \mathrm{hrs}$, but this transfer is reduced to only $5.6 \mathrm{hrs}$ by starting from $r_{0}=10,000 \mathrm{~km}$. The $\Delta v$ associated with these Hohmann transfers is nearly constant and has a weak maximum near $r_{0}=30,000 \mathrm{~km}$. Over the interval $10,000 \mathrm{~km} \leq r_{0} \leq$ $70,000 \mathrm{~km}$, Hohmann transfer $\Delta v$ to zero selenocentric speed at the lunar surface is never more than $2.6 \mathrm{~km} / \mathrm{s}$ nor less than $2.5 \mathrm{~km} / \mathrm{s}$.

The propulsive cost associated with crewed vehicle transfer from Earth's vicinity to an SDRO can be compared as $r_{0}$ is changed. To keep transit time reasonably short, assume this transfer will entail a selenocentric approach asymptotic speed $v_{\infty}=1.0 \mathrm{~km} / \mathrm{s}$. For $r_{0} \geq 12,500 \mathrm{~km}, v_{\infty}=$ $1.0 \mathrm{~km} / \mathrm{s}$ dictates a powered flyby with pericynthion $100 \mathrm{~km}$ above the Moon's surface, followed by orbit circularization at $r_{0}$, will require less total $\Delta v$ to achieve the desired SDRO than would a single impulse at $r_{0}$. Under all these conditions, plus the further assumption of conic selenocentric motion, Table 2 summarizes the decrease in $\Delta v$ to achieve SDRO with increasing $r_{0}$.

Table 2. Two impulses are required to efficiently arrive at SDROs with $r_{0}=12,500 \mathrm{~km}$, $25,000 \mathrm{~km}$, and 70,000 km assuming selenocentric approach asymptotic speed $v_{\infty}=1.0 \mathrm{~km} / \mathrm{s}$ and conic selenocentric motion. The first impulse is assumed to occur at a flyby pericynthion height $100 \mathrm{~km}$ above the lunar surface, establishes apocynthion at $\boldsymbol{r}_{0}$, and has a retrograde magnitude $\Delta v_{F B}$. The second impulse occurs at $r_{0}$, establishes a circular SDRO, and has a prograde magnitude $\Delta v_{C}$. These impulse magnitudes, together with the sum $\Delta v_{T o T}=\Delta v_{F B}+\Delta v_{C}$, are provided for the three SDROs.

\begin{tabular}{|c|c|c|c|}
\hline $\boldsymbol{r}_{\boldsymbol{0}}(\mathbf{k m})$ & $\boldsymbol{\Delta} \boldsymbol{v}_{\boldsymbol{F B}}(\mathrm{km} / \mathbf{s})$ & $\boldsymbol{\Delta} \boldsymbol{v}_{\boldsymbol{C}}(\mathrm{km} / \mathbf{s})$ & $\boldsymbol{\Delta} \boldsymbol{v}_{\boldsymbol{T} \boldsymbol{T} \boldsymbol{T}}(\mathrm{km} / \mathbf{s})$ \\
\hline 12,500 & 0.360 & 0.309 & 0.669 \\
\hline 25,000 & 0.288 & 0.279 & 0.567 \\
\hline 70,000 & 0.237 & 0.205 & 0.442 \\
\hline
\end{tabular}

\footnotetext{
** In return from an SDRO, imposing a $\Delta v$-optimal departure epoch will require transit time variations approaching one day if Earth landing at a specific geographic longitude is required.
} 
Note that Table 2's pedigree makes its $\Delta v$ values equally applicable to efficient SDRO departure. In a departure scenario, the first $\Delta v_{C}$ impulse is retrograde, followed by the prograde $\Delta v_{F B}$ impulse. The propulsive cost of achieving and departing a PSDRO with respect to an $r_{0}=$ $70,000 \mathrm{~km}$ baseline should therefore be considered in the context of mission-specific objectives and relevant PSDRO attributes. When this trade is juxtaposed with an Earth departure $\Delta v$ near 3 $\mathrm{km} / \mathrm{s}$, the extra $0.2 \mathrm{~km} / \mathrm{s}$ to achieve or depart PSDRO may be justifiable.

\section{B. Planar Considerations And Consequences}

A second $\Delta v$ variation in transits from an SDRO to Earth arises when Earth does not lie sufficiently near the SDRO plane at the SDRO departure epoch. In addition to $\Delta v$ variations, this geometry can lead to an additional dedicated plane change impulse associated with SDRO departure. Planar corrections and their $\Delta v$ variations during Earth return can be virtually eliminated if the SDRO is sufficiently co-planar with the Moon's geocentric orbit. This geometry also facilitates time-critical Earth return. Spot-checks of SDRO examples at $r_{0}=$ $12,500 \mathrm{~km}$ and at $r_{0}=70,000 \mathrm{~km}$ indicate a stable SDRO initiated in the Moon's geocentric orbit plane remains within $2^{\circ}$ of that plane throughout a subsequent 100 -year coast. This degree of planar alignment virtually eliminates corresponding Earth return $\Delta v$ variations.

An SDRO co-planar with the Moon's geocentric orbit will have near-equatorial nadir selenographic latitude at any time because lunar equatorial obliquity is $6.67^{\circ}$ (Ref. 8). In a PSDRO, this constraint can limit lunar surface visibility since selenographic inclination will be near $173^{\circ}$. But, even at $r_{0}=12,500 \mathrm{~km}$, all lunar surface locations with selenographic latitude $-53^{\circ}<\phi<+53^{\circ}$ will observe infrastructure in an equatorial SDRO at least $30^{\circ}$ above the local horizontal at its closest approach. At any time, $40 \%$ of a lunar hemisphere observes infrastructure at $r_{0}=12,500 \mathrm{~km}$ more than $30^{\circ}$ above the local horizontal (see Figure 7).

Selenographic motion in a PSDRO is very nearly westward at all times. At $r_{0}=12,500 \mathrm{~km}$, a lunar surface location with $-53^{\circ}<\phi<+53^{\circ}$ will observe PSDRO-resident infrastructure at least $30^{\circ}$ above the local horizontal for 9.7 hours. At $r_{0}=25,000 \mathrm{~km}$, continuous viewing above $30^{\circ}$ increases to 26.9 hours. These protracted communications windows support continuous command and control of surface assets over one or more human duty shifts.

\section{Earth And Sun Eclipses By The Moon}

Figure 7 illustrates eclipses of the Earth and Sun by the Moon about to commence as seen from a PSDRO with $r_{0}=12,500 \mathrm{~km}$. During an Earth eclipse, direct communications with PSDRO-resident infrastructure is not possible. Similarly, solar-powered infrastructure must rely on stored or alternative energy supplies during an eclipse of the Sun. Frequency and duration of these eclipses is therefore to be minimized to the degree higher priorities permit. 


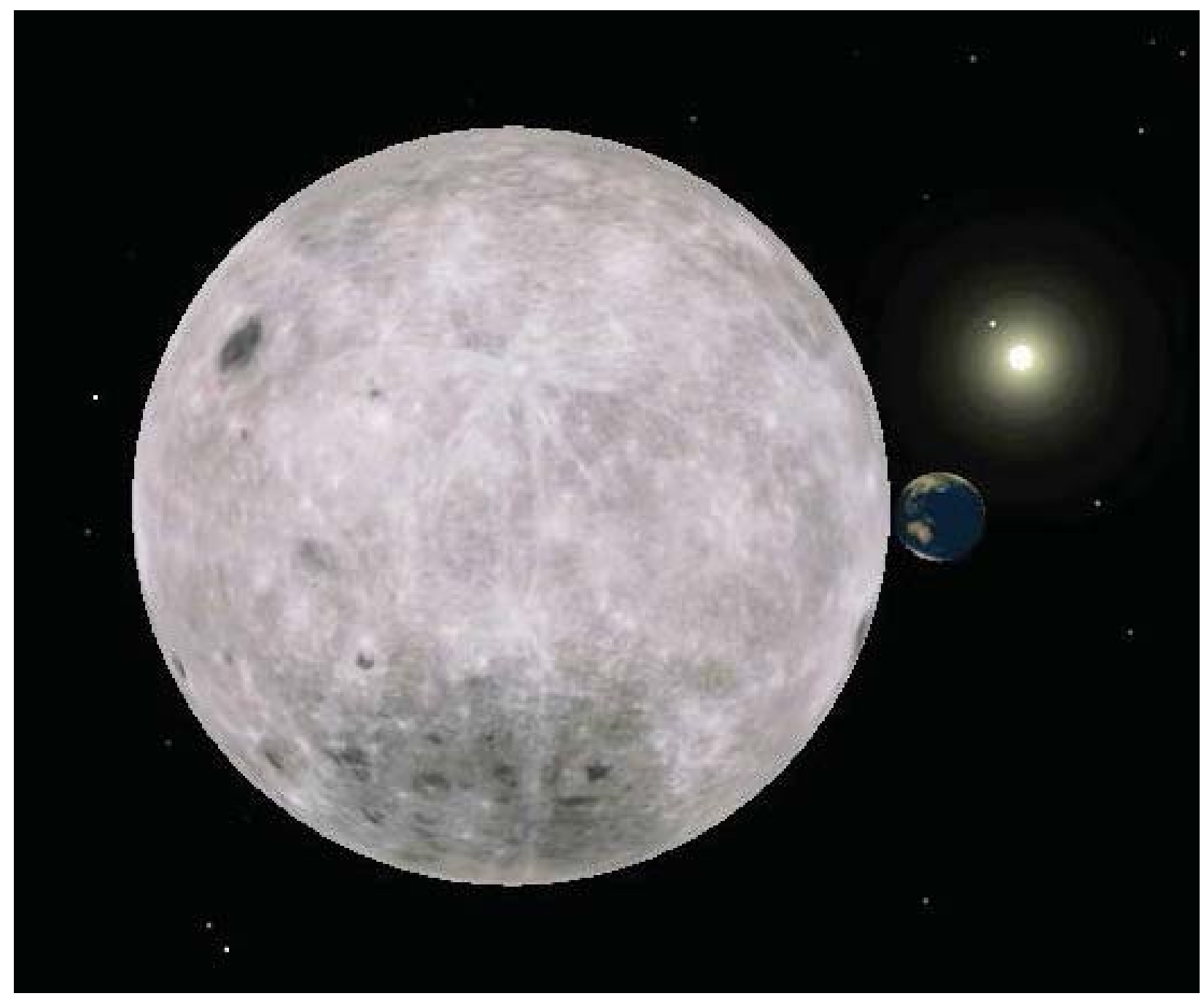

Figure 7. The Earth and Sun are about to undergo eclipses by the Moon as seen from a PSDRO with $r_{0}=12,500 \mathrm{~km}$. This image is rendered by Celestia open-source planetarium software.

Table 3 lists uninterrupted sequences of eclipses as seen from SDROs near the Moon's geocentric orbit plane with $r_{0}=70,000 \mathrm{~km}$ and $r_{0}=12,500 \mathrm{~km}$. Solar eclipses from $r_{0}=70,000$ $\mathrm{km}$ occur less frequently (about once every half year) than at $r_{0}=12,500 \mathrm{~km}$ (about once every 34.6 hours) for two reasons. First, the Moon's apparent diameter is less than $3^{\circ}$ at $r_{0}=70,000$ $\mathrm{km}$ and $16^{\circ}$ at $r_{0}=12,500 \mathrm{~km}$. Second, the Moon's geocentric orbit is inclined $5.145^{\circ}$ with respect to the ecliptic (Ref. 8), the plane to which the Sun's inertial motion is confined. Since both Table 3 SDROs orbit near the plane of lunar motion about Earth, an Earth eclipse occurs every orbit (once every 13 days at $r_{0}=70,000 \mathrm{~km}$, and once every 1.4 days at $r_{0}=12,500 \mathrm{~km}$ ). 
Table 3. Uninterrupted eclipse sequences of the Earth and Sun as observed from SDROs nearly coplanar with the Moon's geocentric orbit are listed for $\boldsymbol{r}_{\boldsymbol{0}}=\mathbf{7 0 , 0 0 0} \mathrm{km}$ and $\boldsymbol{r}_{\boldsymbol{0}}=$ $12,500 \mathrm{~km}$. Eclipse durations are computed from 1st contact (when the Earth or Sun begins disappearing behind the Moon) to 4th contact (when the Earth or Sun finishes reappearing from behind the Moon).

\begin{tabular}{|c|c|c|c|}
\hline $\begin{array}{r}r_{0}=70,000 \mathrm{~km} \\
\text { 1st Contact Year } 2013 \mathrm{UT}\end{array}$ & Duration & $\begin{aligned} r_{0}=12,500 \mathrm{~km} \\
\text { 1st Contact Year } 2013 \mathrm{UT}\end{aligned}$ & Duration \\
\hline Earth 13 JUL 10:10 & $3 \mathrm{~h} 37 \mathrm{~m}$ & Sun 30 JUN $20: 19$ & $1 \mathrm{~h} 23 \mathrm{~m}$ \\
\hline Earth 26 JUL 16:15 & $3 \mathrm{~h} 30 \mathrm{~m}$ & Earth 01 JUL $20: 11$ & $1 \mathrm{~h} 41 \mathrm{~m}$ \\
\hline Earth 08 AUG 16:42 & $3 \mathrm{~h} 35 \mathrm{~m}$ & Sun 02 JUL $06: 56$ & $1 \mathrm{~h} 22 \mathrm{~m}$ \\
\hline Earth 21 AUG 14:31 & $3 \mathrm{~h} 27 \mathrm{~m}$ & Earth 03 JUL 05:16 & $1 \mathrm{~h} 42 \mathrm{~m}$ \\
\hline Earth 03 SEP 21:25 & $3 \mathrm{~h} 36 \mathrm{~m}$ & Sun 03 JUL $17: 32$ & $1 \mathrm{~h} 22 \mathrm{~m}$ \\
\hline Earth 16 SEP 15:33 & $3 \mathrm{~h} 30 \mathrm{~m}$ & Earth 04 JUL 14:24 & $1 \mathrm{~h} 41 \mathrm{~m}$ \\
\hline Earth 29 SEP 18:57 & $3 \mathrm{~h} 36 \mathrm{~m}$ & Sun 05 JUL $04: 08$ & $1 \mathrm{~h} 21 \mathrm{~m}$ \\
\hline Earth 13 OCT $01: 52$ & $3 \mathrm{~h} 32 \mathrm{~m}$ & Earth 05 JUL 23:32 & $1 \mathrm{~h} 41 \mathrm{~m}$ \\
\hline Earth 25 OCT $18: 55$ & $3 \mathrm{~h} 38 \mathrm{~m}$ & Sun 06 JUL $14: 44$ & $1 \mathrm{~h} 20 \mathrm{~m}$ \\
\hline Sun 31 OCT $17: 12$ & $4 \mathrm{~h} 06 \mathrm{~m}$ & Earth 07 JUL $08: 41$ & $1 \mathrm{~h} 41 \mathrm{~m}$ \\
\hline Earth 08 NOV $06: 37$ & $3 \mathrm{~h} 28 \mathrm{~m}$ & Sun 08 JUL $01: 19$ & $1 \mathrm{~h} 20 \mathrm{~m}$ \\
\hline Earth 21 NOV 02:05 & $3 \mathrm{~h} 41 \mathrm{~m}$ & Earth 08 JUL $17: 50$ & $1 \mathrm{~h} 41 \mathrm{~m}$ \\
\hline Earth 04 DEC $00: 01$ & $3 \mathrm{~h} 24 \mathrm{~m}$ & Sun 09 JUL $11: 54$ & $1 \mathrm{~h} 20 \mathrm{~m}$ \\
\hline Earth 17 DEC $07: 35$ & $3 \mathrm{~h} 37 \mathrm{~m}$ & Earth 10 JUL $02: 58$ & $1 \mathrm{~h} 41 \mathrm{~m}$ \\
\hline Earth 30 DEC $00: 30$ & $3 \mathrm{~h} 29 \mathrm{~m}$ & Sun 10 JUL $22: 29$ & $1 \mathrm{~h} 20 \mathrm{~m}$ \\
\hline
\end{tabular}

In general, eclipses in a PSDRO are more frequent than at $r_{0}=70,000 \mathrm{~km}$, but each of these more frequent events is less than half the duration a corresponding eclipse would be at $r_{0}=$ $70,000 \mathrm{~km}$. The 1.4-hour solar eclipses in Table 3 at $r_{0}=12,500 \mathrm{~km}$ are more akin to those routinely managed by International Space Station power systems than is the single 4-hour event at $r_{0}=70,000 \mathrm{~km}$. At $r_{0}=12,500 \mathrm{~km}$, Table 3 eclipses are less than twice the duration of similar events in low lunar orbit, and they occur far less frequently.

\section{Enhanced SDRO Stability With Decreasing $\boldsymbol{r}_{0}$}

Stability assessments at $r_{0}=70,000 \mathrm{~km}$ indicate only a narrow range of initial selenocentric velocities leads to trajectories satisfying this paper's stability criteria for 100 years. Any pair of these stable velocities defines a $\Delta v$ value less than $0.005 \mathrm{~km} / \mathrm{s}$. In accord with the ARM concept, an inert asteroid or bulk sample whose mass exceeds 200 metric tons can be expected to stay in an $r_{0}=70,000 \mathrm{~km}$ SDRO once a velocity in the narrow stability range is attained because its coasted motion is not subject to the influence of appreciable accelerations from non-gravitational forces. Such is not the case for crewed infrastructure subject to forces from overboard gaseous venting, fluid dumping, and propulsive attitude control.

At $r_{0}=12,500 \mathrm{~km}$, circular initial velocity satisfies stability criteria when coasted for 100 years. Furthermore, this PSDRO velocity can be increased by $0.120 \mathrm{~km} / \mathrm{s}$ and still remain stable for 100 years. The resulting elliptical PSDRO has initial selenocentric apsis distances of 30,598 $\mathrm{x} 12,500 \mathrm{~km}$ and evolves to apsis distances of $32,153 \mathrm{x} 12,473 \mathrm{~km}$ after a 100 -year coast. Clearly, an SDRO for crewed infrastructure can be more easily maintained at more proximal selenocentric distances. 


\section{Telerobotic Lunar Surface Operations From PSDRO}

A PSDRO can offer remarkable opportunities for lunar surface telerobotic control. Fulfilling space exploration tasks by telepresence is a concept that is fairly old, but it has generated new enthusiasm (Ref. 9, 10,11) due to advanced telerobotic technologies. Continued advancements in telerobotics are driven by commercial and defense efforts, together with lack of funding for crewed lunar landers and surface habitats. For science, telepresence allows high quality sampling that can take advantage of hyperspectral and microscopic imaging, large mobility range and multiple surrogates at different sites, and dexterity on many scales. These capabilities are not native to a human doing a surface EVA. To the extent it is of value, such telepresence can be conveniently shared with a larger group of observers on Earth. In addition, telepresence hardware can provide a high degree of situation awareness and long duration site occupation, facilitating highly interactive field geology.

The key to operationally effective telepresence is high bandwidth and low communications latency. Human reaction time, which is of order $0.2 \mathrm{~s} \mathrm{(Ref.} \mathrm{12),} \mathrm{is} \mathrm{the} \mathrm{metric} \mathrm{for} \mathrm{successful} \mathrm{real-}$ time human cognition, whether for human-in-the-loop operation or high quality supervisory control. The Earth-Moon two-way latency of 2.6 seconds is decidedly larger than this. Of course, Earth-Mars communication latencies are vastly larger, so it is well understood that onorbit telerobotic control of lunar surface assets at least exercises strategies that will be enormously useful for putting human cognition on the surface of Mars before we actually put humans there.

Of course, the potential for on-orbit telerobotics goes well beyond the Moon and Mars, offering real-time human cognition at destinations that are much less hospitable to astronauts. It should be emphasized that space exploration by telepresence is an explicit example of partnership between human space flight and robotics. In this context, the purpose of human spaceflight is to get humans close enough to allow for real-time functionality.

While low altitude lunar orbits minimize orbit-to-surface control latency, these orbits offer very limited connection times, and would be poorly suited to surface telepresence. Such orbits are generally also very expensive in stationkeeping propellant and are frequently shadowed. Elliptical orbits can be stable and propulsively economical, but they impose dramatically changing control latencies to the surface. It has been suggested that periodic orbits about EML1 or the trans-lunar libration point (EML2) would be advantageous, in that fairly stable light-time limited two-way latencies of 0.4 seconds would offer high quality cognition. Such orbits provide uninterrupted connection to most of one lunar hemisphere (Ref. 13), and relatively low stationkeeping penalties (Ref. 14). The $r_{0}=70,000 \mathrm{~km}$ SDRO proposed for ARM would offer a 14-day orbit around the Moon, and communication latencies of about half a second.

But PSDROs provide for proportionally smaller comm latency to the surface, even well within the $0.2 \mathrm{~s}$ human reaction-time light distance, as well as increased bandwidth. A PSDRO thereby offers real value for quality teleoperation of surface assets from an orbit that is highly stable. That these orbits offer access to both hemispheres instead of just one, as Lagrange point orbits do, can be seen as advantageous to the extent that assets are distributed across the lunar surface. A PSDRO habitat can be used as a supply depot and way station for future crewed lunar surface facilities (Ref. 15), as well as a tank farm for lunar surface in-situ resource utilization (ISRU) products enabling interplanetary voyages. To the extent a facility of increasing mass is envisioned, the stability of these PSDROs is a major asset because stationkeeping propulsion is minimal. 
Communication requirements from PSDRO habitat to lunar surface asset are important to consider. Assuming $1 \mathrm{Mb} / \mathrm{s}$ bandwidth is required for visual situation awareness and surrogate control, a Mars Science Laboratory-variety $35 \mathrm{~W}$ X-band transmitter with a 2-axis controlled microstrip patch antenna and modest $20 \mathrm{~cm}$ receiving antenna in a $r_{0}=20,000 \mathrm{~km}$ PSDRO can do the job well (see Ref. 16 for Lagrange Point link budget). That same system can be used for direct-to-Earth applications, coupled to the much larger Deep Space Network antennae available on the Earth. While an actively pointed high-gain antenna is a concession on a rover that is bouncing across lunar regolith, the beam size is large enough that preserving the link to PSDRO should not be difficult. A luminous beacon in PSDRO is one strategy to achieve this dynamic pointing. To that extent, and in the interest of much higher bandwidths, laser communications could be considered. Compared to low lunar orbit motion, a PSDRO habitat is highly advantageous because it will move across the lunar surface asset's sky very slowly. Of course, the target bandwidth will need to be carefully matched to the task envisioned and the degree of situation awareness required.

The choice of PSDRO $r_{0}$ is a matter of great interest. Obviously, smaller $r_{0}$ offers advantages in communications latency and bandwidth to the surface. Larger orbits offer propulsion economies to locations beyond the Moon, but smaller orbits offer these economies to the lunar surface. Of particular interest are the human factors pertaining to extended telerobotic control. Operational efficiency will be aided by extended connection time, allowing the astronaut controller to get thoroughly comfortable with the situation of a given surrogate, and the surface morphology in its vicinity. It may be simplistic, however, to assume that the orbit needs to be such that a high quality connection is guaranteed for a nominal workshift. The workload on the astronaut controller will certainly be determined in large part by the degree of autonomy of the surface system. That is, real-time human-in-the-loop control should be reserved for tasks that really need it. It is clearly not in the interest of efficient or low-risk operation to have the astronaut controller trying to switch situation awareness between different surrogates at different sites. Such constraints will become evident from analog experiments with space telerobot simulators on the ground, commercial telerobotic applications, and to the extent microgravity and cognitive overloading is a factor, perhaps from experiments on ISS (Ref. 17).

\section{Interplanetary Human Spaceflight Servicing From PSDRO}

The closest stable orbit to Earth from which multiple human interplanetary departures or arrivals can be supported without substantial $\Delta v$ penalties is an SDRO (Ref. 18). Due to reduced selenographic periods, as noted in Subsection A, PSDROs are particularly well suited in this application. Intrinsic stability in PSDROs facilitates sustained orbit maintenance for human infrastructure and interplanetary transports subject to frequent perturbations from dumped consumables, venting, and out-gassing. Depending on the economic viability of ISRU on the Moon and at near-Earth asteroids, refined consumables for interplanetary transport may be easier to obtain from those sources in PSDRO than from Earth.

Human transport arrival at (or departure from) the Moon's vicinity for an interplanetary destination such as Mars entails sufficient geocentric asymptotic speed to warrant a powered Earth flyby at approximately $+400 \mathrm{~km}$ height. The arrival (or departure) asymptote associated with Mars transits is constrained with respect to the Sun/Earth line and local solar time. Powered Earth flyby perigee and the retrograde trans-lunar injection (TLI) impulse on arrival from Mars therefore occur near 8 AM local solar time. Likewise, powered Earth flyby perigee and the prograde trans-Mars injection (TMI) impulse on departure for Mars occur near 8 PM local solar 
time. These powered flyby perigee constraints in turn require the Moon to be about a day after first quarter phase when the transport arrives at the Moon's vicinity post-TLI and about a day after last quarter phase when the transport departs from the Moon's vicinity pre-TMI.

To enforce reasonably short transit times no more than several days between Earth and Moon during an interplanetary arrival or departure, sufficient selenocentric asymptotic speed is imposed warranting a powered lunar flyby at $+100 \mathrm{~km}$ height for $r_{0}>\sim 10,000 \mathrm{~km}$. Consequently, interplanetary arrivals and departures from PSDRO will typically require a human transport undergo powered flybys of both the Earth and Moon. As an example of such a dualflyby trajectory, consider the following sequence of events departing an $r_{0}=12,500 \mathrm{~km}$ PSDRO for Mars.

Table 4. Trans-Earth injection (TEI) and trans-Mars injection (TMI) impulses are summarized as a human transport departs an $r_{0}=12,500 \mathrm{~km}$ PSDRO for Mars. The leftmost column provides the UT of each event in the rightmost column. Values in the $\Delta v$ column are positive for prograde burns and negative for retrograde burns.

\begin{tabular}{|c|c|l|}
\hline Date @ UT & $\boldsymbol{\Delta v}(\mathbf{k m} / \mathbf{s})$ & Event \\
\hline 2026 Nov 01@ 11:41 & -0.109 & TEIa lowers PSDRO pericynthion height to $+4936 \mathrm{~km}$ \\
\hline 2026 Nov 03@ 09:26 & -0.208 & TEIb lowers pericynthion height to $+100 \mathrm{~km}$ \\
\hline 2026 Nov 03@ 16:52 & +0.488 & TEIc departs Moon for Earth \\
\hline 2026 Nov06@ 23:49 & +0.891 & TMI \\
\hline
\end{tabular}

The Table 4 example also illustrates how a fixed UT and selenographic location at the TEIc impulse can be accommodated for arbitrary PSDRO phasing by lowering pericynthion height to an intermediate value with the TEIa impulse. A longer-period SDRO could require a substantially longer time interval between TEIa and TEIc. In extreme cases, a long-period SDRO could require early departure from the Moon and multiple eccentric Earth orbits spanning weeks to properly phase for TMI. Selenocentric and geocentric trajectories associated with Table 4 impulses are plotted with respect to inertial coordinate systems in Figures 8 and 9, respectively. 


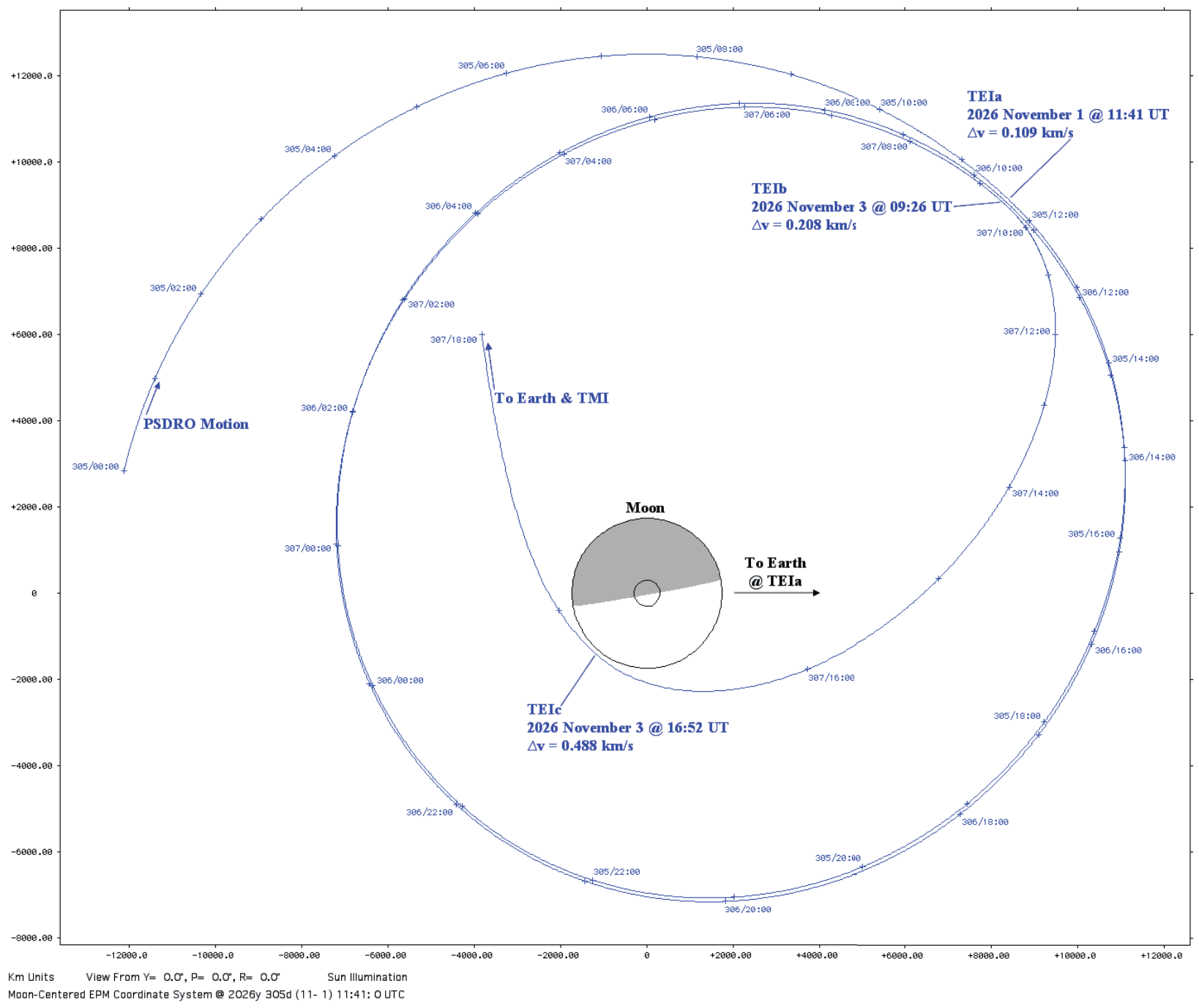

Figure 8. Selenocentric inertial motion of a Mars-bound human transport is plotted as it departs an $r_{0}=12,500 \mathrm{~km}$ PSDRO for Earth and TMI. The plot plane coincides with the Moon's equator and very nearly coincides with that of transport motion. Time tick labels are 2026 November 1-3 UT in day-of-year (DOY)/hh:mm format, and the shaded area is the Moon's nightside.

American Institute of Aeronautics and Astronautics 


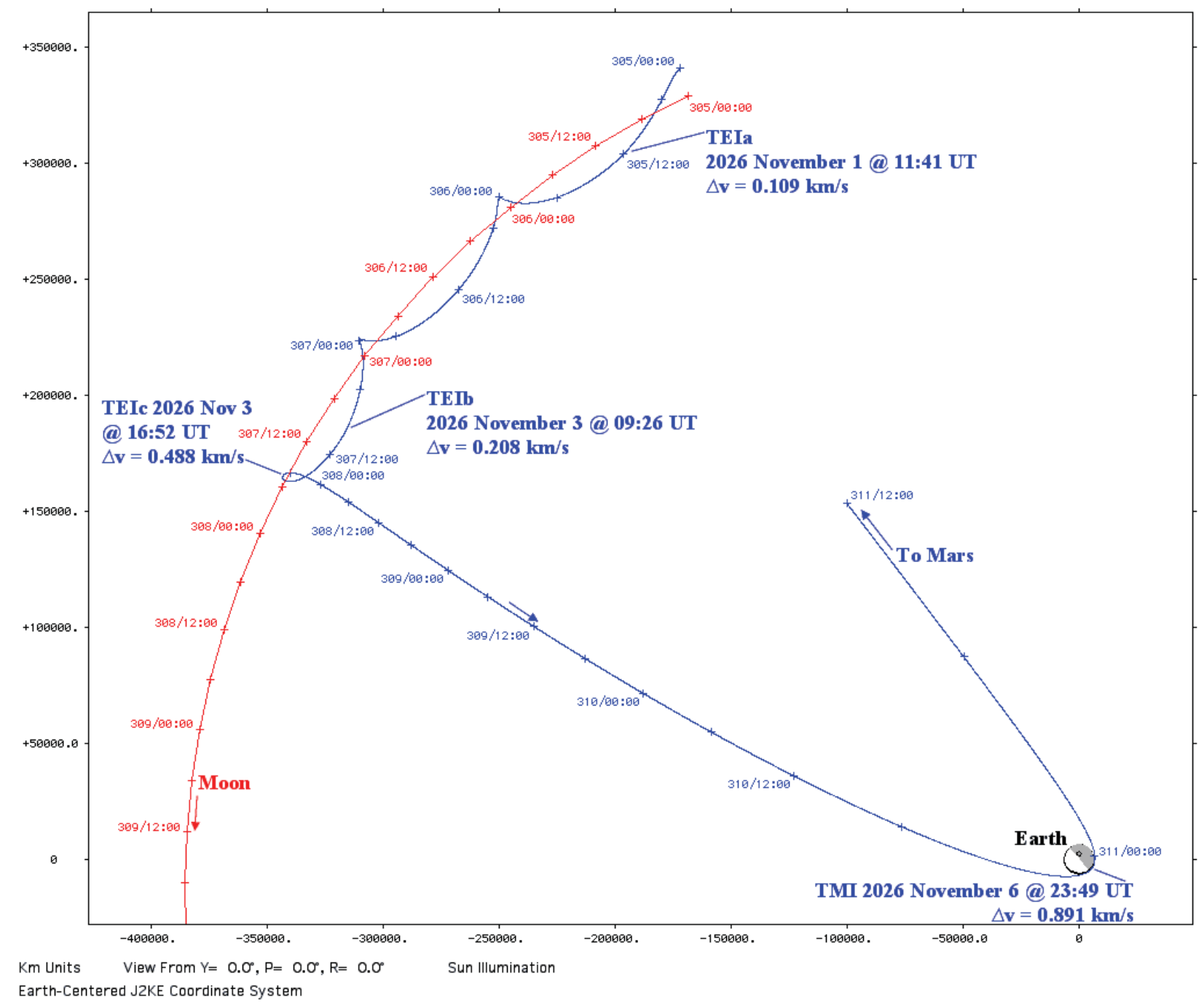

Figure 9. Geocentric inertial motion is plotted for the Moon (red) and a Mars-bound human transport (blue) as it departs the Moon and Earth with powered flybys. The plot plane coincides with the ecliptic plane and very nearly that of transport motion. Time tick labels are 2026 November 1-7 UT in day-of-year (DOY)/hh:mm format, and the shaded area is Earth's nightside. From this shading, note how the Moon must be slightly past last quarter phase at TEIc to minimize TMI $\Delta v$.

\section{Conclusion}

The utility of PSDROs substantially closer to the Moon than the final destination proposed for NASA's ARM payload has been documented in the context of robust human command and control supporting telerobotic lunar surface operations. If such operations extend to near-polar selenographic locations or require continuous human interaction, additional robotic communications infrastructure could be deployed in other PSDROs to act as relays. Even with an additional relay, data latency between command and control infrastructure and surface assets would be less than from ARM's proposed destination SDRO or periodic orbits near the translunar or cislunar libration points.

Any infrastructure deployed in a PSDRO at selenocentric distances from several thousand to $25,000 \mathrm{~km}$ or more enjoys a high degree of stability because the PSDRO plane is near that of the 
Moon's geocentric orbit. Under these conditions, frequent transfers between PSDRO-resident infrastructure and Earth are enabled. This "any-day" flexibility enables routine and contingency logistics between Earth and the infrastructure, together with garaging human interplanetary transports in PSDRO. Direct Earth communications and solar power outages due to eclipses by the Moon in a PSDRO appear to be of longer duration, but at lower frequency, than similar events routinely managed by the International Space Station.

Stability associated with a candidate SDRO will require infrastructure-specific assessment before deployment to include non-gravitational forces beyond the scope of this paper. Particularly for crewed vehicles, such assessments may find the more robust stability margins of PSDROs render them significantly more practical than higher altitude selenocentric orbits.

\section{References}

"NASA's FY2014 Asteroid Strategy", NASA, 2013. ${ }^{\dagger \dagger}$

2"Asteroid Redirect Mission Reference Concept", NASA, 2013."

${ }^{3}$ Johnson Space Center Advanced Mission Design Branch (EG5), "Lunar Mission Tutorial, Part 1 - Lunar Orbit Mechanics", NASA, 2013.

${ }^{4}$ Nesvorný, D., Alvarellos, J. L. A., Dones, L., Levison, H. F., "Orbital And Collisional Evolution Of The Irregular Satellites", The Astronomical Journal, Vol. 126, No. 7, The American Astronomical Society, 2003, pp. 98-429.\$\$

${ }^{5}$ Adamo, D. R., "A Precision Orbit Predictor Optimized For Complex Trajectory Operations," Astrodynamics 2003, Advances in the Astronautical Sciences, Vol. 116, Univelt, San Diego, CA, 2003, pp. 2567-2586.

${ }^{6}$ Roncoli, R.B., Lunar Constants and Models Document, Jet Propulsion Laboratory, 2005. ${ }^{* * *}$

${ }^{7}$ Parker, J. S. and Anderson, R. L., Low-Energy Lunar Trajectory Design, Jet Propulsion Laboratory, 2013.

${ }^{8}$ Giorgini, J. D., Yeomans, D. K., Chamberlin, A. B., Chodas, P. W., Jacobson, R. A., Keesey, M. S., Lieske, J. H., Ostro, S. J., Standish, E. M., and Wimberly, R. N., "JPL's On-Line Solar System Data Service," Bulletin of the American Astronomical Society, Vol. 28, No. 3, 1996, p. $1158 .{ }^{\dagger \dagger}$

${ }^{9}$ International Space Exploration Coordination Group (ISECG), The Global Exploration Roadmap, NP-2013-06-945-HQ, G-28568, NASA, 2013."*

${ }^{10}$ Lester, D., "Putting Human Cognition and Awareness on Other Worlds: A Challenge for Human and Robotic Space Exploration", Space Operations Communicator, April 2014. $\$ \$$

${ }^{11}$ Lester, D., Hodges, K., and Raftery, M. "Telepresence from Orbit", Space News, 2011 .****

\footnotetext{
${ }^{\dagger}$ This document may be downloaded at http://www.nasa.gov/pdf/740684main_LightfootBudgetPresent0410.pdf (accessed 7 Dec 2013).

$\$$ This document may be downloaded at

http://www.nasa.gov/pdf/756122main_Asteroid\%20Redirect\%20Mission\%20Reference\%20Concept\%20Description.pdf (accessed 7 Dec 2013).

$\S \S$ This document may be downloaded at http://www.boulder.swri.edu/ davidn/papers/irrbig.pdf (accessed 10 December 2013 ).

*** This document may be downloaded at http://ssd.jpl.nasa.gov/dat/lunar_cmd_2005_jpl_d32296.pdf (accessed 10 December 2013).

1† This service is documented and available through links at http://ssd.jpl.nasa.gov/?horizons (accessed 14 December 2013 ).

\$+ This document may be downloaded at http://www.globalspaceexploration.org/wordpress/wpcontent/uploads/2013/10/GER 2013.pdf (accessed 5 May 2014).

$\S \S \S$ This document may be downloaded at http:/www.opsjournal.org/assets/SecureDocumentLibrary/DocumentLibraryManager/documents/JSO3-20-14DFLester_final.pdf (accessed 5 May 2014).
} 
${ }^{12}$ Lester, D. and Thronson, H., "Human Space Exploration and Human Spaceflight: Latency and the Cognitive Scale of the Universe", Space Policy, 27, 89.

${ }^{13}$ Grebow, D. J., Ozimek, M. T., and Howell, K. C., "Multibody Orbit Architectures for Lunar South Pole Coverage", Journal of Spacecraft and Rockets, Vol. 45, No. 2, AIAA, March-April 2008, pp. 344-358.

${ }^{14}$ Lester, D. and Thronson, H., "Low-Latency Lunar Surface Telerobotics from Earth-Moon Libration Points", AIAA Space 2011, AIAA 2011-7341.

${ }^{15}$ Garber, S. and Asner, G., "NASA's Decadal Planning Team and the Policy Formulation of the Vision for Space Exploration, NASA, 2009. ${ }^{\dagger \dagger \dagger}$

${ }^{16}$ Lester, D. "Putting Telepresence on the Moon" Lunar Science Forum, 2013.

${ }^{17}$ Garvin, J, Valinia, A., and Thronson, H., "Low Latency Telepresence for Science on the ISS", Technical Interchange Meeting report, NASA, 2013.

${ }^{18}$ Adamo, D. R., "Trajectory Challenges Faced By Reusable Infrastructure In Earth Orbit Supporting Multiple Departures For Mars", The Space Show Classroom, 2013. ${ }^{\$ \S \S}$

\footnotetext{
**** This document may be downloaded at http://www.spacenews.com/article/telepresence-orbit (accessed 5 May 2014).

${ }^{\dagger \dagger \dagger}$ This document may be downloaded at http://history.nasa.gov/DPT/DPT.htm (accessed 5 May 2014).

\$\$ This document may be downloaded at http://telerobotics.gsfc.nasa.gov/papers/ISS_LLT_Science.pdf (accessed 5 May 2014).

$\S \S \S$ This document may be downloaded at http://spaceshowclassroom.files.wordpress.com/2013/11/multiplemarsdeparturesr1.pdf (accessed 23 February 2014).
} 